Original Research Paper

\title{
Free Particle Spin Speed
}

\author{
${ }^{1}$ Nicolae Petrescu and ${ }^{2}$ Florian Ion Tiberiu Petrescu \\ ${ }^{1}$ Bucharest University, Bucharest, (CE), Romania \\ ${ }^{2}$ ARoTMM-IFToMM, Bucharest Polytechnic University, Bucharest, (CE), Romania
}

\author{
Article history \\ Received: 31-05-2019 \\ Revised: 29-06-2019 \\ Accepted: 04-07-2019 \\ Corresponding Author: \\ Florian Ion Tiberiu Petrescu \\ ARoTMM-IFToMM, Bucharest \\ Polytechnic University, \\ Bucharest, (CE), Romania \\ Email: scipub02@gmail.com
}

\begin{abstract}
The electron and the proton are stable particles, compounds of the atoms, which in turn are the macroscopic scale components. A heated metal plate becomes the source of a cloud of electrons, which by an acceleration in an electromagnetic field becomes an electron beam. Protons are obtained by ionized hydrogen atoms; as the mass of the electron is negligibly small relative to the mass of the proton, a hydrogen reservoir is basically a proton reservoir. Many other subatomic particles (elemental or composite) may be produced in elementary processes that occur in nature or in the laboratory. In various physical applications of elementary particles, it is necessary to know the rotation speed around the own axis of an elementary particle in motion. The work determines this angular velocity of rotation of the particle with a very high accuracy depending on its known linear displacement velocity. In other words, the angular velocity of an elementary particle is in the function of its linear displacement velocity, also obtained by De Broglie's hypothesis that the impulse of the elementary particle is preserved. Applications of this new theory can be used in nuclear power, in nuclear fusion and in high-power laser physics. Of the desire to add a new brick to the physical construction of nuclear fusion reactor theory, to obtaining sustainable nuclear energy, but also to the optimal realization and to the other possible applications, one presented this original work.
\end{abstract}

Keywords: Nuclear Fusion, Elementary Particle Dynamics, Condensed Matter, Particle Angular Velocity, Electron Angular Velocity

\section{Introduction}

In particle physics, an elementary particle or fundamental particle is a particle that is not known to have a substructure; this means that it is not known if it is made up of smaller particles. If an elementary particle really does not have any substructure, then it is one of the basic units of the Universe, from which all the other particles are made. In the standard model, elementary particles are fundamental fermions (including quarks, leptons and their antiparticles) and fundamental bosons (including intermediate bosons and Higgs boson).

Though today we consider these particles to be elementary, it is possible that tomorrow they will be removed from this category and find themselves in fact that they can be divided into other smaller particles. That is how it happened in turn with the atom that was originally thought to be an elemental particle, but then it was discovered that it consists of a nucleus and electrons, or of protons and neutrons (nucleons condensed in a nucleus) and electrons. Initially, it was thought that the nucleons are elementary particles, like electrons, but today they are quoted as compounded from quarks, the latter being elemental particles. Here is a big question that no one yet had the courage to put it. If the elementary charge is that of an electron or positron and the quarks have other tasks that cannot be multiples of the elementary load, then either the elementary charge is smaller and the electrons and the quarks are also divided into other smaller particles, or the theory quarks is erroneous, although it has already been verified by several methods today.

The elemental particle physics studies the fundamental constituents of matter and their interactions. An elementary particle is a subatomic particle whose properties can be understood without assuming that it has an internal structure. In the development of quantum physics, some subatomic particles initially considered elementary have subsequently proved to have an internal structure. Exploring the smaller-scale matter structure has been experimentally performed in collision processes with increasing energies: the elemental particle physics is also known as the physics of high energies.

The experimental results obtained and the theoretical models elaborated in the last decades of the 20th century were synthesized in the standard model, according to 
which the elementary particles are the quarks and leptons, together with calibration bosons (which mediate electromagnetic, weak and strong interactions). They are added to the Higgs boson (postulated to explain the different zero of the calibration bosons and experimentally confirmed in 2013). Graviton postulated as a mediator of gravitational interaction has remained hypothetical and is not included in the standard model.

In other words, one can say that some discoveries cancel some older ones, but on the other hand, we are walking on an unknown field, or even more so, with too many unknowns and with too little clear and definitive knowledge.

The electron and the proton are stable particles, compounds of the atoms, which in turn are the macroscopic scale components. A heated metal plate becomes the source of a cloud of electrons, which by acceleration in an electromagnetic field becomes an electron beam. Protons are obtained by ionized hydrogen atoms; as the mass of the electron is negligibly small relative to the mass of the proton, a hydrogen reservoir is basically a proton reservoir. Many other subatomic particles (elemental or composite) may be produced in elementary processes that occur in nature or in the laboratory.

High energy particles from sources located in the alien space produce uninterrupted cascades of secondary particles in the upper layers of the atmosphere. This cosmic radiation is a natural source of particles with far higher energies than those that can be made in a terrestrial laboratory; the positron, the muon, the pion and the kaon were discovered in this way. The energy obtained by such particles has the disadvantage that it is uncontrollable and the particle flow is very low; in addition, cosmic ray experiments require a lot of patience and last a long time.

Nuclear reactions produce a variety of particles, mainly neutrons, neutrinos, electrons and positrons, photons (gamma radiation). Research reactors serve as sources of particulate matter in laboratory experiments. Elementary particles are produced in the laboratory as fragments resulting from collision processes. Subatomic scale fragmentation and massive fragments production require high energies, which are achieved by acceleration. An ion source produces electrically charged particles (protons or electrons); they are injected into the accelerator, where they are accelerated in an electromagnetic field and collimated into a beam that is focused on a target. The collision disintegration products are captured and analyzed by a detector, or filtered and routed into secondary beams.

The particles moving on a closed trajectory emit electromagnetic radiation (synchrotron radiation), which means deceleration and loss of energy in the collision process. For equal energies, the loss is more pronounced in the case of electrons than for protons: linear accelerators (linac) are preferred instead of circular accelerators to accelerate electrons.

Nuclear fusion is the combination of two light nuclei in a heavier nucleus. Fusion or thermonuclear reactions of light elements are typical reactions that occur in the Sun and other stars. Indeed, in the Sun, every second, 657 million tons of hydrogen is converted into 653 million tons of helium. The 4 million tonnes missing are then converted to radiation - this phenomenon assuring the sun's shine. Extreme and high-pressure temperatures create a strongly ionized state of matter, called plasma, which must be maintained in that volume by means of electromagnetic forces (Halliday and Robert, 1966; Kramer, 2011; Krane, 1987; Moses et al., 2009; Petrescu and Calautit, 2016a-b; Petrescu and Petrescu, 2014; 2019; Petrescu et al., 2016; Petrescu, 2012a-b; 2014; 2019; Shultis and Faw, 2002).

A fusion reaction in which a relatively large amount of energy $(27.7 \mathrm{MeV})$ is released is one in which four protons interact leading to the formation of a helium nucleus (an alpha particle). Because hydrogen isotopes are used in this process and hydrogen is virtually all around us, the idea of getting energy from its fusion is extremely attractive: it basically provides an unlimited source of energy for future generations!

In addition, the nuclear fusion reaction uses nonradioactive materials, which are not radioactive (as happens in the nuclear fission reaction), not to mention that everything that results from the fusion reaction is only energy and inert helium gas is very environmentally friendly. The controlled nuclear fusion reaction once started, is easy to control and maintain, not getting out of control as it did in some fission reactors. The price of fuel used is very low and the fact that it is easy to obtain today helps to repeat the reaction to infinity, making controlled nuclear fusion energy an energy-efficient, friendly, sustainable, renewable, green, cheap and compact inexhaustible source.

In all these processes it is necessary to know the basic parameters of the physical particles (elementary or not), including their dimensions and the speeds with which they move and rotate around their own axis (Halliday and Robert, 1966; Kramer, 2011; Krane, 1987; Moses et al., 2009; Petrescu and Calautit, 2016a-b; Petrescu and Petrescu, 2014; 2019; Petrescu et al., 2016; Petrescu, 2012a-b, 2014; 2019; Shultis and Faw, 2002).

\section{Materials and Methods}

Pulse of a particle in linear moving is written using relation (1) (The Lorentz relationship (2) was used to express the mass of the particle):

$p=m \cdot v=\frac{m_{0} \cdot c \cdot v}{\sqrt{c^{2}-v^{2}}}$ 
$m=\frac{m_{0} \cdot c}{\sqrt{c^{2}-v^{2}}}$

The wavelength associated with the particle can be determined with the relationship (3) (according to Louis de Broglie the pulse is conserved):

$\lambda=\frac{h}{p}=\frac{h \cdot \sqrt{c^{2}-v^{2}}}{m_{0} \cdot c \cdot v}$

Wave frequency associated with the particle is determining by relationship (4):

$$
\gamma=\frac{c}{\lambda}=\frac{c \cdot m_{0} \cdot c \cdot v}{h \cdot \sqrt{c^{2}-v^{2}}}=\frac{m_{0} \cdot c^{2} \cdot v}{h \cdot \sqrt{c^{2}-v^{2}}}
$$

The angular velocity of the particle and its square can be calculated with the relationships (5):

$$
\left\{\begin{array}{l}
\omega=2 \pi \gamma=\frac{2 \pi \cdot m_{0} \cdot c^{2} \cdot v}{h \cdot \sqrt{c^{2}-v^{2}}} \\
\omega^{2}=\frac{4 \pi^{2} \cdot m_{0}^{2} \cdot c^{4} \cdot v^{2}}{h^{2} \cdot\left(c^{2}-v^{2}\right)}
\end{array}\right.
$$

It is noted that the angular velocity of a physical particle is dependent on its linear velocity and three physical constants: $m_{0}$ (the resting mass of the particle), $c$ (the velocity of the light in vacuum) and $h$ (the Plank constant).

If one express with beta the ratio between the linear velocity of the particle and the speed of the light considered in the vacuum (6), the expressions (5) take the forms (7):

$$
\beta=\frac{v}{c}
$$

$$
\left\{\begin{array}{l}
\omega=\frac{2 \pi \cdot m_{0} \cdot c^{2} \cdot \beta}{h \cdot \sqrt{1-\beta^{2}}}=\frac{2 \pi \cdot m_{0} \cdot c^{2}}{h} \cdot \frac{\beta}{\sqrt{1-\beta^{2}}} \\
\omega^{2}=\frac{4 \pi^{2} \cdot m_{0}^{2} \cdot c^{4} \cdot \beta^{2}}{h^{2} \cdot\left(1-\beta^{2}\right)}=\frac{4 \pi^{2} \cdot m_{0}^{2} \cdot c^{4}}{h^{2}} \cdot \frac{\beta^{2}}{\left(1-\beta^{2}\right)}
\end{array}\right.
$$

\section{Results and Discussion}

The free proton angular speed in function of $\beta$ can be seen in the Table 1 , in the Table 2 one may see the same parameter (angular velocity) for a free deuteron and in the Table 3 can be followed these parameters (angular velocities) for a free triton particle.

Obviously in this case is about the kinetic energy at rotation around its own axis, but the phenomenon is also true for the kinetic energy in the translational movement of the particles.
It will still try to follow the same parameter in the case of a much lighter particle, the electron (Table 4).

In this case, when the electron is much easier, spin speeds drop considerably compared to the heaviest proton particle.

\section{Conclusion}

Of the desire to add a new brick to the physical construction of nuclear fusion reactor theory, to obtaining sustainable nuclear energy, but also to the optimal realization and to the other possible applications, one presented this original work.

A fusion reaction in which a relatively large amount of energy (27.7 MeV) is released is one in which four protons interact leading to the formation of a helium nucleus (an alpha particle). Because hydrogen isotopes are used in this process and hydrogen is virtually all around us, the idea of getting energy from its fusion is extremely attractive: it basically provides an unlimited source of energy for future generations!

In addition, the nuclear fusion reaction uses nonradioactive materials, which are not radioactive (as happens in the nuclear fission reaction), not to mention that everything that results from the fusion reaction is only energy and inert helium gas is very environmentally friendly. The controlled nuclear fusion reaction once started, is easy to control and maintain, not getting out of control as it did in some fission reactors. The price of fuel used is very low and the fact that it is easy to obtain today helps to repeat the reaction to infinity, making controlled nuclear fusion energy an energy-efficient, friendly, sustainable, renewable, green, cheap and compact inexhaustible source.

In all these processes it is necessary to know the basic parameters of the physical particles (elementary or not), including their dimensions and the speeds with which they move and rotate around their own axis.

The free proton angular speed in function of $\beta$ can be seen in the Table 1 , in the Table 2 one may see the same parameter (angular velocity) for a free deuteron and in the Table 3 can be followed these parameters (angular velocities) for a free triton particle.

Obviously in this case is about the kinetic energy at rotation around its own axis, but the phenomenon is also true for the kinetic energy in the translational movement of the particles.

It will still try to follow the same parameter in the case of a much lighter particle, the electron (Table 4).

In this case, when the electron is much easier, spin speeds drop considerably compared to heavy proton particle.

It can easily be observed that at higher masses of the particle and the rotational speeds around its own axis are increasing, which can be explained by the fact that a heavier particle has higher kinetic energy than an easier one. 
Table 1: The free proton angular speed in function of $\beta$

\begin{tabular}{llll}
\hline$\beta$ & 0.000009 & 0.00002 & 0.0001 \\
$\omega\left[\mathrm{s}^{-1}\right]$ & $1.28 \mathrm{E}+19$ & $2.85 \mathrm{E}+19$ & $1.43 \mathrm{E}+20$ \\
$\beta$ & 0.001 & 0.01 & 0.1 \\
$\omega\left[\mathrm{s}^{-1}\right]$ & $1.43 \mathrm{E}+21$ & $1.43 \mathrm{E}+22$ & $1.43 \mathrm{E}+23$ \\
$\beta$ & 0.2 & 0.3 & 0.4 \\
$\omega\left[\mathrm{s}^{-1}\right]$ & $2.91 \mathrm{E}+23$ & $4.48 \mathrm{E}+23$ & $6.22 \mathrm{E}+23$ \\
$\beta$ & 0.5 & 0.6 & 0.7 \\
$\omega\left[\mathrm{s}^{-1}\right]$ & $8.23 \mathrm{E}+23$ & $1.07 \mathrm{E}+24$ & $1.40 \mathrm{E}+24$ \\
$\beta$ & 0.8 & 0.9 & 0.99 \\
$\omega\left[\mathrm{s}^{-1}\right]$ & $1.90 \mathrm{E}+24$ & $2.94 \mathrm{E}+24$ & $1.00 \mathrm{E}+25$ \\
$\beta$ & 0.999 & 0.9999 & 0.99999 \\
$\omega\left[\mathrm{s}^{-1}\right]$ & $3.19 \mathrm{E}+25$ & $1.01 \mathrm{E}+26$ & $3.19 \mathrm{E}+26$ \\
$\beta$ & 0.999999 & 0.9999999 & 0.99999999 \\
$\omega\left[\mathrm{s}^{-1}\right]$ & $1.01 \mathrm{E}+27$ & $3.19 \mathrm{E}+27$ & $1.01 \mathrm{E}+28$ \\
\hline
\end{tabular}

Table 2: The free deuteron angular speed in function of $\beta$

\begin{tabular}{llll}
$\beta \beta$ & 0.000009 & 0.00002 & 0.0001 \\
$\omega\left[\mathrm{s}^{-1}\right]$ & $2.565 \mathrm{E}+19$ & $5.701 \mathrm{E}+19$ & $2.850 \mathrm{E}+20$ \\
$\beta$ & 0.001 & 0.01 & 0.1 \\
$\omega\left[\mathrm{s}^{-1}\right]$ & $2.850 \mathrm{E}+21$ & $2.850 \mathrm{E}+22$ & $2.864 \mathrm{E}+23$ \\
$\beta$ & 0.2 & 0.3 & 0.4 \\
$\omega\left[\mathrm{s}^{-1}\right]$ & $5.818 \mathrm{E}+23$ & $8.964 \mathrm{E}+23$ & $1.244 \mathrm{E}+24$ \\
$\beta$ & 0.5 & 0.6 & 0.7 \\
$\omega\left[\mathrm{s}^{-1}\right]$ & $1.645 \mathrm{E}+24$ & $2.137 \mathrm{E}+24$ & $2.794 \mathrm{E}+24$ \\
$\beta$ & 0.8 & 0.9 & 0.99 \\
$\beta\left[\mathrm{s}^{-1}\right]$ & $3.800 \mathrm{E}+24$ & $5.885 \mathrm{E}+24$ & $2.000 \mathrm{E}+25$ \\
$\omega\left[\mathrm{s}^{-1}\right]$ & 0.999 & 0.9999 & 0.99999 \\
$\beta$ & $6.369 \mathrm{E}+25$ & $2.015 \mathrm{E}+26$ & $6.373 \mathrm{E}+26$ \\
$\omega\left[\mathrm{s}^{-1}\right]$ & 0.999999 & 0.9999999 & 0.99999999 \\
\hline
\end{tabular}

Table 3: The free triton angular speed in function of $\beta$

\begin{tabular}{|c|c|c|c|}
\hline$\beta$ & 0.000009 & 0.00002 & 0.0001 \\
\hline$\omega\left[\mathrm{s}^{-1}\right]$ & $3.841 \mathrm{E}+19$ & $8.537 \mathrm{E}+19$ & $4.268 \mathrm{E}+20$ \\
\hline$\beta$ & 0.001 & 0.01 & 0.1 \\
\hline$\omega\left[\mathrm{s}^{-1}\right]$ & $4.268 \mathrm{E}+21$ & $4.268 \mathrm{E}+22$ & $4.290 \mathrm{E}+23$ \\
\hline$\beta$ & 0.2 & 0.3 & 0.4 \\
\hline$\omega\left[\mathrm{s}^{-1}\right]$ & $8.713 E+23$ & $1.342 \mathrm{E}+24$ & $1.862 \mathrm{E}+24$ \\
\hline$\beta$ & 0.5 & 0.6 & 0.7 \\
\hline$\omega\left[\mathrm{s}^{-1}\right]$ & $2.464 \mathrm{E}+24$ & $3.201 E+24$ & $4.184 \mathrm{E}+24$ \\
\hline$\beta$ & 0.8 & 0.9 & 0.99 \\
\hline$\omega\left[\mathrm{s}^{-1}\right]$ & $5.69 \mathrm{E}+24$ & $8.813 E+24$ & $2.995 E+25$ \\
\hline$\beta$ & 0.999 & 0.9999 & 0.99999 \\
\hline$\omega\left[\mathrm{s}^{-1}\right]$ & $9.53 \mathrm{E}+25$ & $3.018 \mathrm{E}+26$ & $9.544 \mathrm{E}+26$ \\
\hline$\beta$ & 0.999999 & 0.9999999 & 0.99999999 \\
\hline$\omega\left[\mathrm{s}^{-1}\right]$ & $3.018 \mathrm{E}+27$ & $9.544 \mathrm{E}+27$ & $3.018 \mathrm{E}+28$ \\
\hline$\beta$ & 0.000009 & 0.00002 & 0.0001 \\
\hline$\omega\left[\mathrm{s}^{-1}\right]$ & $6.987 \mathrm{E}+15$ & $1.552 \mathrm{E}+16$ & $7.764 \mathrm{E}+16$ \\
\hline$\beta$ & 0.001 & 0.01 & 0.1 \\
\hline$\omega\left[\mathrm{s}^{-1}\right]$ & $7.764 \mathrm{E}+17$ & $7.764 \mathrm{E}+18$ & $7.803 \mathrm{E}+19$ \\
\hline$\beta$ & 0.2 & 0.3 & 0.4 \\
\hline$\omega\left[\mathrm{s}^{-1}\right]$ & $1.584 \mathrm{E}+20$ & $2.44 \mathrm{E}+20$ & $3.388 \mathrm{E}+20$ \\
\hline$\beta$ & 0.5 & 0.6 & 0.7 \\
\hline$\omega\left[\mathrm{s}^{-1}\right]$ & $4.48 \mathrm{E}+20$ & $5.82 \mathrm{E}+20$ & $7.61 \mathrm{E}+20$ \\
\hline$\beta$ & 0.8 & 0.9 & 0.99 \\
\hline$\omega\left[\mathrm{s}^{-1}\right]$ & $1.03 \mathrm{E}+21$ & $1.60 \mathrm{E}+21$ & $5.44 \mathrm{E}+21$ \\
\hline$\beta$ & 0.999 & 0.9999 & 0.99999 \\
\hline$\omega\left[\mathrm{s}^{-1}\right]$ & $1.73 \mathrm{E}+22$ & $5.48 \mathrm{E}+22$ & $1.73 E+23$ \\
\hline$\beta$ & 0.999999 & 0.9999999 & 0.99999999 \\
\hline$\omega\left[\mathrm{s}^{-1}\right]$ & $5.49 \mathrm{E}+23$ & $1.73 E+24$ & $5.49 \mathrm{E}+24$ \\
\hline
\end{tabular}




\section{Acknowledgement}

This text was acknowledged and appreciated by Dr. Veturia CHIROIU Honorific member of Technical Sciences Academy of Romania (ASTR) Ph.D. supervisor in Mechanical Engineering.

\section{Funding Information}

Research contract: Contract number 36-5-4D/1986 from 24IV1985, beneficiary CNST RO (Romanian National Center for Science and Technology) Improving dynamic mechanisms internal combustion engines:

1. New Aircraft (New Ionic or Beam Engines): no. 548 of 22-04-2010 [cgiywDssin], Aerospace Engineering

2. Some Few Specifications About the Doppler Effect to the Electromagnetic Waves: 636 of 28-05-2010 [iEtcaouxxA], physics

3. Presenting an Atomic Model and Some Possible Applications in LASER Field: nr. 639 of 29-052010 [yncngrotfo], physics

4. Some Applications in LASER Field: no. 718 of 0907-2010 [xeujouincC], physics

5. The Energies of Today and Tomorrow: nr. 819 of 30-09-2010 [kbHquxwykr], energy engineering

6. Obtaining Energy by the Annihilation of the Matter with Antimatter - The Battle for Energy: nr. 1068 of 13.03.2011 [GfEqpGDzeh], Energy Engineering

\section{Author's Contributions}

All the authors contributed equally to prepare, develop and carry out this manuscript.

\section{Ethics}

This article is original and contains unpublished material. Authors declare that are not ethical issues and no conflict of interest that may arise after the publication of this manuscript.

\section{References}

Halliday, D. and R. Robert, 1966. Physics, Part II. 1st Edn., John Wiley and Sons, Inc., New York.

Kramer, D., 2011. DOE looks again at inertial fusion as a potential clean-energy source. Phys. Today, 64: 26-26. DOI: 10.1063/1.3563814
Krane, K.S., 1987. Introductory Nuclear Physics. 3rd Edn., Wiley and Sons, New York, ISBN-10: 047180553X, pp: 864.

Moses, EI., R.N. Boyd, B.A. Remington, C.J. Keane and R. Al-Ayat, 2009. The national ignition facility: Ushering in a new age for high energy density science. Phys. Plasmas, 16: 041006-041006. DOI: $10.1063 / 1.3116505$

Petrescu, F.I.T. and J.K. Calautit, 2016a. About nano fusion and dynamic fusion. Am. J. Applied Sci., 13: 261-266. DOI: 10.3844/ajassp.2016.261.266

Petrescu, F.I.T. and J.K. Calautit, 2016b. About the light dimensions. Am. J. Applied Sci., 13: 321-325. DOI: 10.3844/ajassp.2016.321.325

Petrescu, F.I.T. and R.V., Petrescu, 2014. Nuclear green energy, Int. J. Am. Phys., 10: 3-14.

Petrescu, F.I.T. and R.V.V. Petrescu, 2019. Nuclear hydrogen structure and dimensions. Int. J. Hydrogen Energy, 44: 10833-10837. DOI: 10.1016/J.IJHYDENE.2019.02.140

Petrescu, F.I.T., A. Apicella, R.V. Petrescu, S. Kozaitis and R. Bucinell et al., 2016. Environmental protection through nuclear energy. Am. J. Applied Sci., 13: 941-946. DOI: 10.3844/ajassp.2016.941.946

Petrescu, F.I.T., 2014. Nuclear fusion. Infinite Energy, 20: 44-47.

Petrescu, F.I.T., 2012a. Cold nuclear fusion. Plasma Phys. Fus. Technol.

Petrescu, F.I.T., 2012b. Cold Nuclear Fusion. 1st Edn., Create Space Publisher, USA, ISBN-10: 978-14782-3426-5, pp: 80.

Petrescu, F.I.T., 2019. About the nuclear particles' structure and dimensions. Comp. Part. Mech., 6: 191-194. DOI: 10.1007/S40571-018-0206-7

Shultis, JK. and R.E. Faw, 2002. Fundamentals of nuclear science and engineering. 1st Edn., CRC Press, New York, ISBN-10: 0824708342, pp: 520.

\section{Nomenclature}

$\mathrm{h}=>$ the Planck constant: $\mathrm{h}=6.626 \mathrm{E}-34$ [Js]

$\mathrm{q}=>$ electrical elementary load: $\mathrm{q}_{\mathrm{e}}=-1.6021 \mathrm{E}-19[\mathrm{C}]$

$\mathrm{q}_{\mathrm{p}}=+1.6021 \mathrm{E}-19[\mathrm{C}]$

$\mathrm{c}=$ the light speed in vacuum: $\mathrm{c}=2.997925 \mathrm{E}+08[\mathrm{~m} / \mathrm{s}]$

$\mathrm{m}_{0}[\mathrm{~kg}]=>$ the rest mass of one particle

$\mathrm{m}_{0 \text { electron }}=9.11 \mathrm{E}-31[\mathrm{~kg}]$

$\mathrm{m}_{\text {0proton }}=1.672621898(21) \mathrm{E}-27[\mathrm{~kg}]$

$\mathrm{m}_{\text {0neutron }}=1.674927471(21) \mathrm{E}-27[\mathrm{~kg}]$

$\mathrm{m}_{\text {0deuteron }}=3.34449 \mathrm{E}-27[\mathrm{~kg}]$

$\mathrm{m}_{\text {Otriton }}=5.00827 \mathrm{E}-27[\mathrm{~kg}]$ 